Didáctica. Lengua y literatura

ISSN-e: $1988-2548$

\title{
La evaluación de herramientas como modo de aprendizaje e introducción de las Humanidades Digitales en el aula universitaria. La experiencia docente "Poesía distante"1
}

\author{
Clara I. Martínez Cantón²; Pablo Ruiz Fabo ${ }^{3}$
}

Fecha recepción: 29-01-2018 / Fecha aceptación: 07-03-2019

Resumen. Las tecnologías y su dimensión ubicua se hacen presentes en todas las disciplinas y han cambiado la forma de estudiar e investigar, creando campos como las Humanidades Digitales cuyo auge es ya innegable. Los estudios de grado en humanidades no siempre reflejan este giro digital. En este artículo presentamos los resultados de una experiencia docente cuyo objetivo ha sido la mejora de la adquisición de los contenidos de métrica en poesía del grado, así como la adquisición de contenidos transversales en relación a la tecnología y la digitalidad. Para ello se ha combinado la enseñanza de la métrica española con la de las tecnologías computacionales que se aplican a su análisis desde las Humanidades Digitales, buscando una implicación directa de los estudiantes en la investigación universitaria. Con ese fin se organizó una actividad que planeó videoconferencias con expertos internacionales y a una actividad de anotación métrica de sonetos para su procesado computacional. Los resultados, obtenidos por medio de una encuesta de satisfacción y las calificaciones de los estudiantes, muestran una clara mejora en los resultados de la asignatura para los que participaron en esta experiencia y una alta satisfacción con los contenidos referentes a tecnología y la digitalidad aprendidos. Ciertos aspectos como el tamaño de la muestra o la necesidad de otros datos comparativos quedan para la discusión de esta experiencia.

Pablabras clave: Humanidades Digitales, Métrica, Enseñanza online, Herramientas literarias digitales, Webconferencias.

\section{[en]Literary Tool Evaluation as a Way of Introducing Digital Humanities in University Courses. The "Distant Poetry" case}

\footnotetext{
Abstract. Technologies, with their ubiquitous dimension, have presence in all disciplines and have changed the way of studying and researching in the humanities, with fields such as Digital Humanities whose relevance is undeniable. Nevertheless, Humanities degrees in Spanish universities do not always reflect this digital turn. This paper presents the results of a teaching innovation activity. Its goal was to improve the acquisition of poetic metrics and the acquisition of transversal contents related to technology and digitality in a Modern Languages (Spanish) degree. The activity combined the teaching of Spanish metrics and of the computational technologies used for its automatic analysis in Digital Humanities projects, trying to involve the students in university research. The activity consisted in webconferences with international experts, and a task where the students were asked to metrically

1 Este trabajo se enmarca dentro de la Actividad de Innovación Docente (AIE) de la UNED: "Poesía distante: el análisis métrico, hacia su automatización y evaluación".

2 Departamento de Literatura Española y Teoría de la Literatura, UNED

cimartinez@flog.uned.es

3 Laboratoire LiLPa, Université de Strasbourg

ruizfabo@unistra.fr
} 
annotate sonnets, knowing that their annotation would be used to test a system for automatic metrical analysis. Results were measured by two means: a satisfaction form filled by students, and their marks in the course. Results show a clear improvement for those students that joined the activity and a high satisfaction with the technological and digital contents that they learnt. Some issues, such as sample size or the need of more data to compare are discussed.

Keywords: Digital Humanities, Metrics, Distant Online Teaching, Literary Digital Tools, Webconferences.

\section{[fr] L'évaluation des outils littéraires comme moyen d'introduire les humanités numériques dans les cours universitaires. L'expérience d'enseignement "Poésie à distance"}

Résumé. L'omniprésence des technologies dans toutes les disciplines scientifiques a changé la façon dont nous menons nos activités de recherche, en créant des domaines comme les Humanités numériques, qui connaissent un essor indéniable. Or, les programmes en Sciences humaines à l'université espagnole ne reflètent pas toujours ce tournant numérique. Dans cet article, nous présentons les résultats d'une expérience d'innovation pédagogique. Son objectif était d'améliorer l'acquisition de contenus en métrique poétique, ainsi que l'acquisition de contenus transversaux sur la technologie et le numérique, dans un programme de licence en Études hispaniques. À cette fin, l'activité a combiné l'enseignement de la métrique espagnole et des technologies computationnelles utilisées pour l'analyse métrique automatique en Humanités numériques, en cherchant une implication directe des étudiants dans la recherche. L'activité comportait d'une part des conférences web avec des experts internationaux. D'autre part, une tâche où les étudiants ont effectué une annotation métrique manuelle de sonnets, sachant que leurs annotations seraient utilisées pour tester un système d'analyse métrique automatique, en comparant les résultats manuels et automatiques. L'expérience d'innovation pédagogique a été évaluée par deux moyens: Premièrement, à travers une enquête de satisfaction remplie par les étudiants. Deuxièmement, selon les notes obtenues par les étudiants dans le cours. L'enquête a montré une grande satisfaction concernant les contenus reliés à la technologie et aux méthodes numériques abordés dans cette expérience. Les notes des étudiants ayant participé à l'expérience ont été nettement meilleures que celles du reste des étudiants. Nous discutons également des questions comme la taille de l'échantillon ou le besoin de plus de données comparatives.

Mots clés: Humanités numériques, métrique (poésie), enseignement en ligne, outils littéraires numériques, conférences web.

Sumario: 1. Introducción. 2. Estado del arte. Métrica y su análisis computacional en Humanidades Digitales. 3. La experiencia docente. El aprendizaje de Humanidades Digitales a través de la anotación de un corpus de sonetos. 3.1. Contexto y metodología. 3.2. Experiencia "poesía distante". Descripción y actividades. .2.1. El corpus de sonetos y su anotación. 3.2.2. Programa de charlas y debate con expertos. 3.3. Evaluación de la experiencia docente. 4. Resultados. 4.1. Resultados obtenidos del cuestionario a los estudiantes. 4.2. Resultados obtenidos teniendo en cuenta las calificaciones finales. 5. Discusión. 6. Conclusiones. 7. Bibliografía

Cómo citar: Martínez Cantón, C. I.; Ruiz Fabo, P. (2019). La evaluación de herramientas como modo de aprendizaje e introducción de las Humanidades Digitales en el aula universitaria. La experiencia docente "Poesía distante", Didáctica. Lengua y literatura, 31, 171-190

\section{Introducción}

La actual cibersociedad se caracteriza por la ubicuidad de la digitalidad, que ha cambiado el mundo y también, consecuentemente, el modo en el que entendemos las humanidades, impactando directamente tanto en la investigación como en la edu- 
cación. Una revisión de los trabajos más relevantes a este respecto nos llevaría a publicaciones fundacionales del ámbito como A Companion to Digital Humanities (Schreibman, Siemens, y Unsworth, 2004) y el concepto revisitado recientemente en A New Companion to Digital Humanities (Schreibman, Siemens, y Unsworth, 2016). Para una revisión del impacto de las Humanidades Digitales en la investigación humanística cabe citar el libro Debates in the Digital Humanities (Gold, 2012). En el ámbito hispánico Elena González-Blanco (2013) y Antonio Rojas (2013), entre otros, recogen una situación de la investigación humanística con métodos digitales constantemente en movimiento, y que avanza imparablemente. Además, la Revista de Humanidades Digitales (UNED, CONICET y UNAM, s.f.), que en 2019 ha publicado ya su tercer número, ofrece un amplio panorama de la investigación en Humanidades Digitales (en adelante HD) en el ámbito hispánico y su gran empuje como disciplina.

Sin embargo, en España, los programas y cursos de HD no son todavía demasiado abundantes. La situación está, no obstante, cambiando rápidamente. Desde el curso 2016-2017 la Universidad Pablo de Olavide ofrece un máster oficial en Historia y Humanidades Digitales, y en ese mismo curso la Universidad Complutense de Madrid estrenó su Máster en Letras Digitales. Existe un Máster en Humanidades Digitales en la UNED que funciona como título propio desde este 2019 y encontramos además muchos otros títulos propios en universidades como Salamanca o la Universidad Autónoma de Barcelona. La Universidad de Castilla-La Mancha tuvo en su momento uno de los primeros títulos propios de esta disciplina.

No existen títulos de grado en Humanidades Digitales, pero las llamadas competencias digitales sí son consideradas casi siempre como un área de conocimiento transversal. La LOMCE (2013) - Ley orgánica para la mejora de la calidad educativa- en su currículo para Primaria, ESO y Bachillerato señala la adquisición de la competencia digital como una de las siete competencias básicas que debe adquirir el alumnado antes de su ingreso en la universidad. También han tomado relieve las 1lamadas competencias blandas o soft skills referidas a destrezas instrumentales, emocionales, cognitivas y digitales, entre otras.

El caso que nos ocupa es el programa de Grado de Lengua y Literatura Españolas de la UNED, la universidad con mayor número de alumnos en España (156000 en 2015-2016 según el MECD), cuya enseñanza se cursa a distancia. En este programa de grado no hay ninguna asignatura específica que cubra la enseñanza de competencias digitales aplicadas a las humanidades. Las competencias dedicadas a la tecnología aparecen en el apartado de competencias transversales en la memoria de verificación, y son las siguientes: "CT2A4 - Comunicación y expresión matemática, científica y tecnológica (cuando sea requerido y estableciendo los niveles oportunos); CT2B1 - Manejo de las TIC" (UNED, 2014).El Espacio Europeo de Educación Superior (EEES), con su enfoque por competencias reabrió el debate sobre las competencias transversales (Mir Acebrón, 2008, p. 1), especialmente porque estas apuntan en muchos casos a la necesidad de profesionalizar la formación universitaria, acercándola al mercado laboral. Se aboga por la introducción de las tecnologías digitales que parece responder a esta necesidad. Sin embargo, en el aula universitaria, y en especial en los grados más humanísticos, no ha mejorado la competencia digital, ni pura ni blanda desde que en 2007 se enunciara "si nos centramos en la universidad española, el uso y desarrollo de las nuevas tecnologías en la enseñanza parece limitado actualmente a determinadas experiencias, o a ciertas asignaturas que requieren el empleo de las mismas" (Maroto Sánchez, 2007, p. 62). 
Este artículo presenta una experiencia de introducción de las HD, de su definición, de su alcance, de las actividades y de los métodos, en la asignatura universitaria "Estilística y métrica españolas" del grado de Lengua y Literatura españolas de la UNED. Las actividades tecnológicas programadas y llevadas a cabo en esta experiencia funcionaron de manera optativa, reforzando las competencias transversales del grado. En ella se ofreció a los estudiantes la posibilidad de participar en la anotación métrica digital de un amplio corpus de sonetos, introduciéndoles mediante charlas y debates en las nociones fundamentales de las Humanidades Digitales e informándoles de las actividades de investigación y evaluación de herramientas digitales que eran posibles gracias a la anotación del corpus en el que estaban trabajando.

Esta experiencia responde también a una necesidad de enseñar habilidades y métodos de investigación (competencia general CGINS07 de la memoria de la UNED) a los estudiantes, poniendo en comunicación el mundo docente con los proyectos de investigación y la digitalización.

\section{Estado del arte. métrica y su análisis computacional en humanidades digitales}

La naturaleza eminentemente formal del análisis métrico lo hace un campo propicio para su tratamiento computacional en proyectos de HD (Birnbaum y Thorsen, 2015; Delente y Renault, 2015; Río Riande, González-Blanco, y Martínez Cantón, 2015). En los últimos años han ido apareciendo varios proyectos cuyo objetivo es el estudio y la compilación de datos y obras sobre la métrica que aprovechan para este tipo de trabajo las tecnologías digitales. La web como posibilidad para acceder $\mathrm{u}$ ofrecer distintos tipos de recursos hace que estos proyectos ganen en difusión y usabilidad.

El Laboratorio de Innovación en Humanidades Digitales (LINHD) de la UNED ha realizado mucho trabajo en lo referente a métrica y a las propias HD. Funciona como observatorio y cruce de caminos de investigadores en el que la colaboración y el trabajo conjunto hacen posible el nacimiento de nuevos proyectos, en ocasiones complementarios, ofreciendo formación y experiencia (Gonzalez-Blanco, Martínez Cantón y Río Riande, 2015).

En el campo filológico la creación y puesta en abierto de ediciones digitales es quizás uno de los campos más relevantes que han tenido lugar. En el caso de la métrica la creación de bases de datos, y de repertorios poéticos y métricos en versión digital hace que contemos con varios proyectos de relevancia. En la propia UNED se han desarrollado los proyectos ReMetCa (2012), "Repertorio Digital de Poesía Europea" (2015-2017), y la ERC POSTDATA (2016-2021) liderados por Elena González-Blanco o DISCO, un corpus de sonetos españoles analizados métricamente (Ruiz Fabo, Bermúdez Sabel, Martínez Cantón, González-Blanco, y Navarro Colorado, 2018). Es un fenómeno que se da para todas las literaturas europeas, con bases de datos de poesía en múltiples idiomas, que permiten búsquedas métricas y otras interesantes funciones. Entre ellas destacamos en francés: Nouveau Naetebus (Le Noveau Naetebus, s.f.), en italiano: Bibliografia Elettronica dei Trovatori (BedT) (Asperti y De Nigro, 2012), para el húngaro: Répertoire de la poésie hongroise ancienne (RPHA) (Horváth, 2001), para el latín: Corpus Rhythmorum Musicum (Stella y Barret, s.f.), Annalecta Hymnica Digitalia (Rauner, s.f.),Pedecerto (Colombi, Mondini, Tessarolo y Bacianini, s.f.), en gallego-portugués: Cantigas de Santa María (Parkinson, s.f.), Base de Datos da Lírica profana galego-portuguesa 
(MedDB2)(Brea y Lorenzo Grandin, s.f.), en castellano: Repertorio Métrico Digital de la Poesía Medieval Castellana (ReMetCa) (González-Blanco, s.f.), en holandés: Dutch Song Database (Nederlandse Liederenbank, s.f.), para el occitano: The last song of the Troubadours (The Last Song of the Troubadours, s.f.), en escáldico: The Skaldic Project (Wills, s.f.), para el alemán: Lyrik des Minnesänger (Braun, Glauch y Kragl, s.f.), y para el inglés: Digital Edition of the index of Middle English Verse (Digital Index of Middle English Verse, s.f.), Eighteenth-Century Poetry Archive (ECPA) (Huber, s.f.), Cantum pulcriorem invenire: Thirteenth-Century Music and Poetry (University of Southampton, s.f.), entre otros.

En esta experiencia que vamos a presentar nos centraremos sobre todo en la aplicación digital del procesamiento del lenguaje natural (PLN) a la métrica. El PLN ofrece grandes posibilidades para el análisis métrico, ya que permite el análisis y extracción automática de grandes cantidades de información de un corpus de textos a través de reglas definidas según criterios lingüísticos. Ya existen muchas tecnologías útiles para el estudio de la métrica de la desde este punto de vista, partiendo, generalmente, de un enfoque lingüístico, más que métrico. Las más relevantes son aquellas dedicadas a la lematización, el análisis morfológico y de constituyentes y dependencias sintácticas. Para el español específicamente cabe destacar los kits de herramientas Freeling (Lloberes, Castellón, y Padró, 2010; Padró y Stanilovsky, 2012), Apertium (Forcada et al., 2011), IXA Pipes (Agerri, Bermudez, y Rigau, 2014) o las herramientas del IULA-UPF-CLARIN.

Centrándonos exclusivamente en automatización de análisis en métrica española, la investigación más relevante son los estudios en escansión silábica y detección de acentos realizados por Navarro Colorado (Navarro-Colorado, Lafoz, y Sánchez, 2016) y Navarro Colorado (2017), Agirrezabal (2017) y Gervás (2000a). Asimismo, los trabajos en generación automática de poesía que sigue patrones métricos de Gervás (Gervás, 2000b, 2015).

\section{La experiencia docente. el aprendizaje de humanidades digitales a través de la anotación de un corpus de sonetos}

\subsection{Contexto y metodología}

El fin buscado con esta investigación ha sido aplicar y evaluar una experiencia innovadora que combinara la enseñanza de la métrica española con la de las tecnologías computacionales que se aplican a su análisis desde las Humanidades Digitales, buscando una implicación directa de los estudiantes.

Como hemos expuesto, esta experiencia docente se llevó a cabo en la asignatura de "Estilística y métrica española" del grado en Lengua y Literatura españolas de la UNED en el curso 2016-2017 (optativa de primer cuatrimestre, 5 créditos ECTS). El perfil de los estudiantes era el de personas sin ninguna experiencia en los métodos y actividades de las Humanidades Digitales, de edades variadas y con formaciones previas diversas. La gran mayoría de los estudiantes pertenecían al segundo curso del grado. En esta asignatura había 199 matriculados, de los que solo 137 se presentaron al examen. El número de estudiantes que siguió la actividad de innovación digital docente fue de 85. Esta experiencia ha buscado añadir un aspecto activo y colaborativo a la asignatura en la que ha sido puesta en marcha. 
La evaluación de esta actividad se llevó a cabo mediante dos herramientas: una encuesta de satisfacción cuya validez fue contrastada con expertos en diseño experimental y en TIC y, por otra parte, con los resultados en las calificaciones. Una vez terminados los cursos, se entregó un cuestionario con 39 ítems a los alumnos. Se les pidió que respondieran de forma voluntaria, y lo hicieron 25 de un total de 85 estudiantes que siguieron la actividad de innovación docente basada en las herramientas de automatización digital de análisis métrico. Se evaluaron también las calificaciones de los estudiantes que siguieron y de los que no siguieron la asignatura.

El objetivo general de la experiencia de innovación docente ha sido la mejora de la adquisición de los contenidos de la asignatura (la métrica y el encabalgamiento), así como la formación en la investigación en Humanidades digitales y, por último la adquisición de contenidos transversales en relación a la tecnología usada y a la digitalidad.

Los objetivos específicos propuestos han sido los siguientes:

- Mejorar la comprensión de los contenidos de la asignatura, en especial el tema de la pausa métrica y el encabalgamiento.

- Mejorar la motivación y el interés de los estudiantes por la asignatura.

- Abordar mediante actividades específicas la adquisición de competencias transversales relacionadas con la tecnología.

- Transmitir los últimos avances en el área de conocimiento de la asignatura.

- Formar en nuevos métodos y perspectivas de investigación en Humanidades útiles para el Grado.

\subsection{Experiencia "Poesía distante". Descripción y actividades}

La experiencia docente "Poesía distante"4 se asentó principalmente en dos tipos de actividades:

1. Webconferencias motivadoras con expertos investigadores de Humanidades Digitales y Procesamiento del Lenguaje Natural. Los ponentes introdujeron a los estudiantes en el mundo de los métodos y herramientas de las HD utilizados en el ámbito de la poesía y de la métrica, sobre las que versa la asignatura.

2. Anotación manual de un corpus de sonetos, anotando las siguientes características: escansión silábica, acentuación del verso y encabalgamiento. Los poemas anotados han sido utilizados para la evaluación de herramientas digitales de análisis métrico y de análisis del encabalgamiento. Las herramientas digitales creadas han sido evaluadas y son presentadas por sus propios creadores en las webconferencias que reciben los estudiantes.

Esta experiencia docente busca hacer converger la enseñanza, la tecnología y la investigación, introduciendo a los estudiantes en la realización de una investigación en HD y utilizando los resultados obtenidos con su corpus manualmente anotado para evaluar herramientas digitales.

$4 \quad$ Esta experiencia toma el nombre del conocido libro de Franco Moretti Distant Reading (2013), fundamental en un nuevo tipo de análisis literario frecuentemente adoptado en las Humanidades Digitales. 


\subsubsection{El corpus de sonetos y su anotación}

El corpus está basado en dos colecciones públicas de antologías de sonetos, disponibles a través de la Biblioteca Virtual Cervantes (García González, 2006b, 2006a). La primera colección recoge 1088 sonetos de 477 autores del siglo XV al XVII y la segunda recoge 2673 sonetos de 685 autores del siglo XIX. Para preparar el corpus se hicieron scripts de bajada de los poemas, para limpiar el código HTML y para extraer las fechas de nacimiento y muerte de los autores. ${ }^{5}$ Además, se desarrolló un script para crear, para cada poema, una plantilla para la anotación (instrumento de trabajo) de cada una de las características métricas que nos interesaban, tal y como se muestra en la Figura 1.

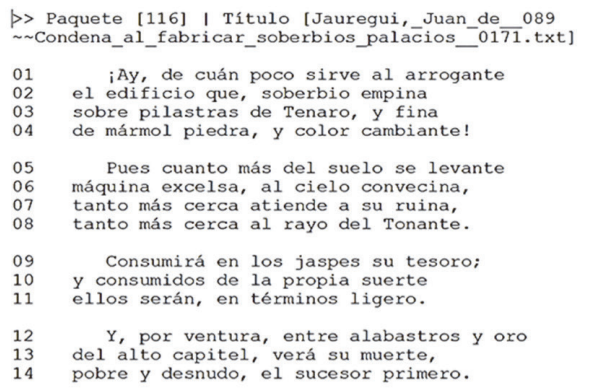

\begin{tabular}{llll}
$>$ & \multicolumn{3}{c}{ ENCABALGAMIENTOS } \\
01 & 02 & $\mathrm{~A}[\mathrm{~B}$ & $\mathrm{B}[]$ \\
02 & 03 & $\mathrm{~A}[\mathrm{~B}$ & $\mathrm{B}[]$ \\
03 & 04 & $\mathrm{~A}[\mathrm{~B}$ & $\mathrm{B}[]$ \\
04 & 05 & $\mathrm{~A}[\mathrm{~B}$ & $\mathrm{B}[]$ \\
05 & 06 & $\mathrm{~A}[\mathrm{~B}$ & $\mathrm{B}[]$ \\
06 & 07 & $\mathrm{~A}[\mathrm{~B}$ & $\mathrm{B}[]$ \\
07 & 08 & $\mathrm{~A}[\mathrm{~B}$ & $\mathrm{B}[]$ \\
08 & 09 & $\mathrm{~A}[]$ & $\mathrm{B}[]$ \\
09 & 10 & $\mathrm{~A}[]$ & $\mathrm{B}[]$ \\
10 & 11 & $\mathrm{~A}[]$ & $\mathrm{B}[]$ \\
11 & 12 & $\mathrm{~A}[]$ & $\mathrm{B}[]$ \\
12 & 13 & $\mathrm{~A}[]$ & $\mathrm{B}[]$ \\
13 & 14 & $\mathrm{~A}[]$ & $\mathrm{B}[]$
\end{tabular}
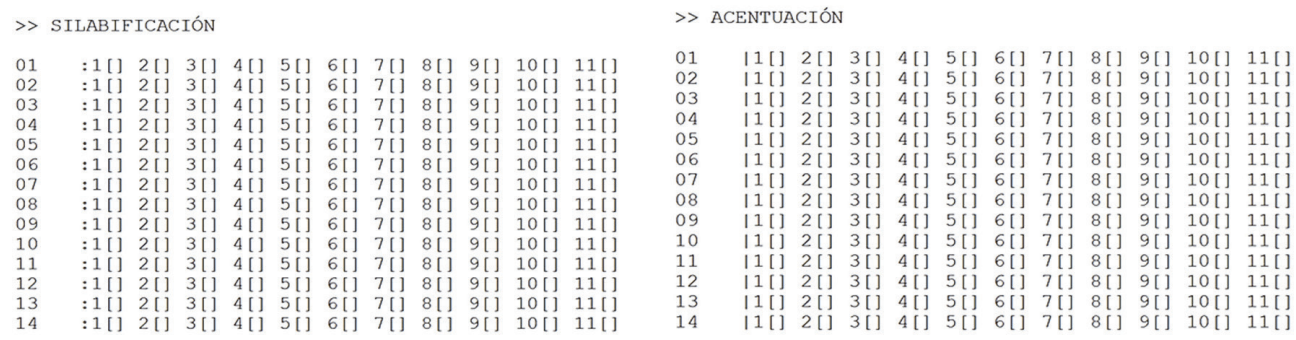

Fig 1. Plantilla para la anotación de sonetos

A los alumnos se les propuso esta actividad de análisis métrico como Prueba de Evaluación continua voluntaria, en la que cada uno debía analizar métricamente, según la plantilla y la guía propuesta, un total de 6 sonetos.

Para realizar la anotación se dio a los estudiantes una Guía de anotación muy detallada y un videotutorial para la realización de la anotación. La guía, el videotutorial y una una carpeta de muestra de seis sonetos puede ser consultados en el siguiente sitio: https://sites.google.com/site/spanishenjambment/dh-intro-throughmetrics.

En resumen la actividad consistió en realizar un análisis métrico de seis sonetos distintos para poder evaluar las herramientas digitales y comprobar su eficacia. Debían marcar la división en sílabas de cada verso, su acentuación métrica y la existencia o no de encabalgamiento entre versos y su tipo. Este análisis métrico contribuiría

5 El corpus está disponible públicamente en GitHub, (https://github.com/pruizf/disco), en versión texto plano y también en versión XML/TEI con metadatos detallados. 
a afianzar los conocimientos de la asignatura y, adicionalmente, serviría para crear un corpus de prueba para evaluar herramientas informáticas de análisis automático.

\subsubsection{Las herramientas que se evaluaban}

Los estudiantes saben en todo momento, gracias a las webconferencias realizadas por expertos, que su anotación de sonetos dará lugar a un corpus anotado manualmente que serviría para la evaluación de dos herramientas diferentes:

a. Herramienta de escansión silábica y detección de acento versal en endecasílabos (Navarro-Colorado, 2017) (la herramienta ya ha sido anteriormente evaluada).

b. Una nueva herramienta desarrollada en 2017 (Ruiz, Martínez Cantón, Poibeau, y González-Blanco, 2017) diseñada para detectar automáticamente encabalgamientos y sus tipos en poesía española.

\subsubsection{Programa de charlas y debate con expertos}

Las charlas han sido llevadas a cabo por expertos internacionales en Humanidades Digitales en cada charla: Gimena del Río Riande (CONICET, Argentina), Borja Navarro (Universidad de Alicante), Pablo Ruiz Fabo (LINHD, UNED) y Clara Martínez Cantón (UNED). Las charlas se encuentran disponibles online en la web institucional de la UNED, Cadena Campus: https://www.intecca.uned.es/portalavip/ emisiones.php?termino=versificaci $\% \mathrm{~F} 3 \mathrm{n}$

El programa ha sido planificado desde un enfoque primero más general e introductorio para pasar posteriormente a temáticas concretas sobre métrica y herramientas digitales de análisis automático. Así, se abordaron la introducción a las HD, qué es el PLN, la aplicación de estos métodos y herramientas a la poesía, hasta la presentación de cómo se evalúan las herramientas utilizando un corpus anotado manualmente, y los resultados de esas herramientas.

Los ponentes en cada una de las webconferencias fueron: Gimena del Río Riande, Borja Navarro, Pablo Ruiz Fabo y Clara Martínez Cantón. Las charlas se encuentran disponibles online en la web institucional de la UNED, Cadena Campus: https:// www.intecca.uned.es/portalavip/emisiones.php?termino=versificaci $\% F 3 n$

\subsection{Evaluación de la experiencia docente}

Hemos querido evaluar la experiencia docente conforme a la siguiente escala:

Tabla 1. Categorías, criterios e indicadores de la evaluación

\begin{tabular}{|l|l|l|}
\hline \multicolumn{1}{|c|}{ Categoría } & \multicolumn{1}{|c|}{ Criterios } & \multicolumn{1}{c|}{ Indicadores } \\
\hline 1. Planificación & Adecuación & Información sobre la actividad de innovación \\
\hline & Satisfacción & Organización de la actividad de innovación \\
\hline 2. Desarrollo & Adecuación & Concordancia con la planificación \\
\hline
\end{tabular}




\begin{tabular}{|c|l|l|}
\hline \multicolumn{1}{|c|}{ Categoría } & \multicolumn{1}{|c|}{ Criterios } & \multicolumn{1}{|c|}{ Indicadores } \\
\hline & Satisfacción & Aspectos didácticos \\
\hline & Satisfacción & Aspectos relacionales \\
\hline 3. Resultados & Adecuación & Tasa mínima de resultados de aprendizaje \\
\hline & Satisfacción & Satisfacción del alumnado con la actividad docente \\
\hline $\begin{array}{r}\text { 4. Orientación a } \\
\text { la innovación }\end{array}$ & Eficiencia & Contribución al aprendizaje y la motivación del alumnado \\
\hline & Satisfacción & Pentenidos innovadores \\
\hline
\end{tabular}

Los instrumentos utilizados para evaluar estas dimensiones distintas marcadas en la escala han sido:

- Un cuestionario de satisfacción (disponible en https://goo.gl/forms/To3i3hwzpKv0aZHS2). Cada indicador se concretaba con una serie de descriptores específicos para las charlas y para la actividad de marcado.

- Las calificaciones de los estudiantes de la asignatura, tratadas de manera conjunta como datos estadísticos.

Respecto al cuestionario de satisfacción, la validez de constructo se ha conseguido mediante la validación de tres expertos, dos profesoras de la Universidad de León del área de Didáctica y una profesora de la UNED de filología, con los criterios de pertinencia, relevancia y univocidad, puntuados conforme a una escala de cuatro niveles para cada uno de los criterios. Los ítems se han mantenido cuando al menos dos expertos puntuaban más de dos y se han modificado en caso contrario. El cuestionario de 48 ítems originales quedó reducido a 39 después de la validación.

Además hemos evaluado la experiencia teniendo en cuenta los resultados obtenidos en la nota final de la asignatura, que se decide en base a un examen. Hemos hecho un estudio que analiza los resultados finales en la asignatura de los estudiantes que realizaron la actividad de innovación "Poesía distante" en comparación con la de los que no la realizaron.

\section{Resultados}

\subsection{Resultados obtenidos del cuestionario a los estudiantes}

La dispersión de los estudiantes por edad es muy grande, con estudiantes desde los 24 a los 68 años. La edad más frecuente es 52 años, la media de edad está en 44,32 años, mientras que la mediana ha sido de 44,5 . Este dato es llamativo y mucho más alto que en cualquier otra investigación con estudiantes universitarios de un centro presencial. La naturaleza de universidad a distancia de la UNED, en la que muchos estudiantes trabajan a la vez que estudian, y realizan en ocasiones su segunda carrera explica estos datos. 
Como puede verse en la Fig.2 los estudiantes están matriculados en diversos cursos, pero el curso con mayor tasa de frecuencia es $2 .^{\circ}$, lo cual es un indicativo de que su formación filológica, tratándose de una asignatura, además, de primer cuatrimestre, no sea todavía muy alta.

\section{Curso del grado en el que está matriculado}

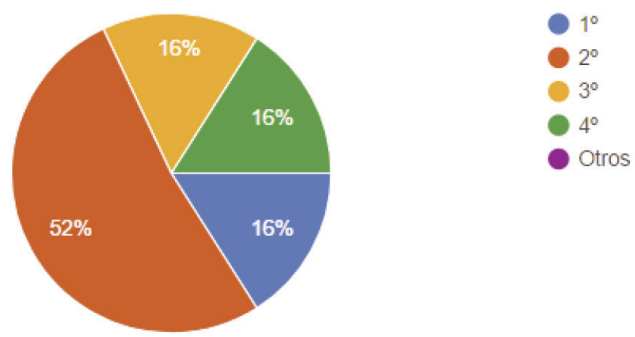

Fig 2. Curso del grado en el que están los estudiantes

El formulario de satisfacción con el que se ha evaluado la experiencia docente consta de 5 categorías acordes con lo expuesto en la tabla 1 más una extra que explicaremos a continuación. Las cuatro propuestas en la tabla han sido: planificación, desarrollo, resultados y orientación a la innovación. La categoría extra se refiere a la consideración de los estudiantes sobre los encabalgamientos, los criterios teóricos tenidos en cuenta para describirlos y su efecto estilístico. Esta categoría es importante más para la evaluación de la herramienta de investigación que detecta los encabalgamientos que para la experiencia docente en sí misma.

La primera categoría es la referente a la planificación de la experiencia docente. En este apartado había un total de 13 preguntas valoradas en una escala del 1-10. Los estudiantes no estaban obligados a puntuar cada uno de los ítems, por lo que dejaron algunos sin puntuar, lo cual ha incidido en una menor puntuación en algunos de ellos, que no indica que su puntuación sea baja por aquellos que la puntuaron. Por este motivo hemos realizado el análisis y las gráficas con los valores de media de cada ítem:

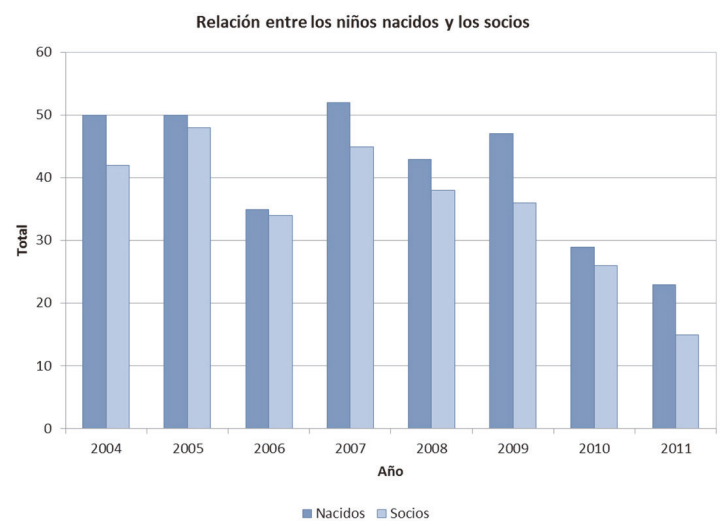

Fig 3. Satisfacción con la planificación de la experiencia docente 
La planificación tiene una valoración muy positiva, destacando el ítem "El profesor cumple con los plazos establecidos para la actividad de innovación docente", con una puntuación media de 9,4 puntos y 25 respuestas. La nota menor en los ítems obtenida ha sido de una media de 8,1 , en la pregunta que evaluaba la planificación de la actividad: "Sobre la 1PEC: El sistema de codificación para anotar la escansión silábica es sencillo". En este apartado la nota individual más baja ha sido de 5, pero encontramos, en contraposición, una frecuencia muy alta de puntuación de 10.

La segunda categoría, en la que los estudiantes evaluaban el desarrollo de la experiencia docente "Poesía distante", constaba asimismo de 13 ítems que los estudiantes valoraban del 1-10. Los resultados fueron los siguientes, centrándonos de nuevo en el análisis de los valores promedio:

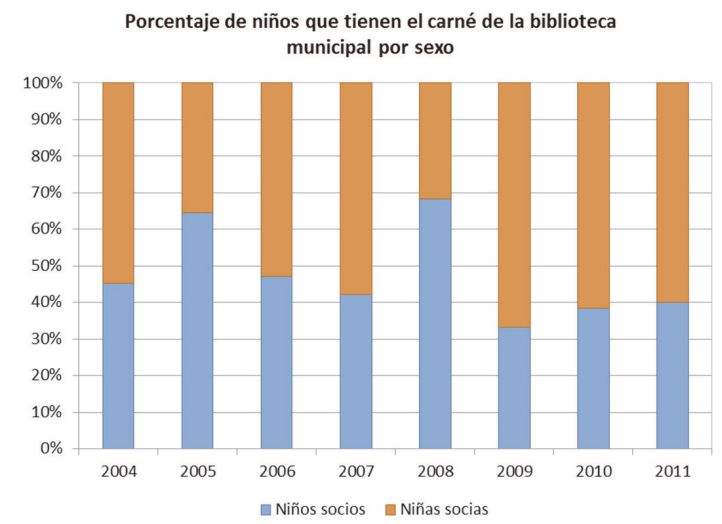

Fig 4. Satisfacción con el desarrollo de la experiencia docente

Los valores obtenidos son también muy positivos, destacando especialmente los tres ítems que valoraban al profesor y su actuación en la resolución de dudas, con puntuaciones mayores a 9,2. El ítem peor valorado ha sido "Me he informado y sentido más involucrado en las actividades de investigación universitaria gracias a esta actividad", si bien la puntuación es de 8,08.

En la categoría quizás más relevante, la dedicada a los resultados, solamente había 4 ítems:

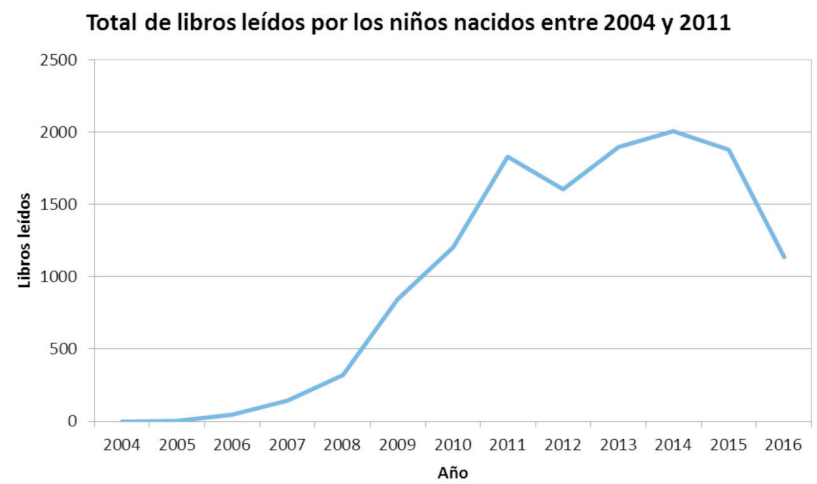

Fig 5. Satisfacción con los resultados de la experiencia docente 
Los resultados, si bien han sido positivos, no son tan buenos como en las categorías anteriores, lo que resulta llamativo tratándose precisamente de la percepción que los alumnos han tenido sobre los resultados. El ítem con menor puntuación es el referente a: "En general: Esta actividad ha despertado más mi interés por la asignatura", con una nota promedio de 8,33. La más alta ha sido la dedicada a las charlas por webconferencia realizadas por expertos sobre Humanidades Digitales que roza casi el 8,7. Consideran, no obstante, que tanto las charlas como la actividad de evaluación continua (1PEC) han mejorado sus conocimientos y competencias en la materia.

En la categoría de orientación a la innovación se han propuesto en el cuestionario para su valoración también 4 ítems, en los que se valoraba el grado de innovación de la actividad y su utilidad:

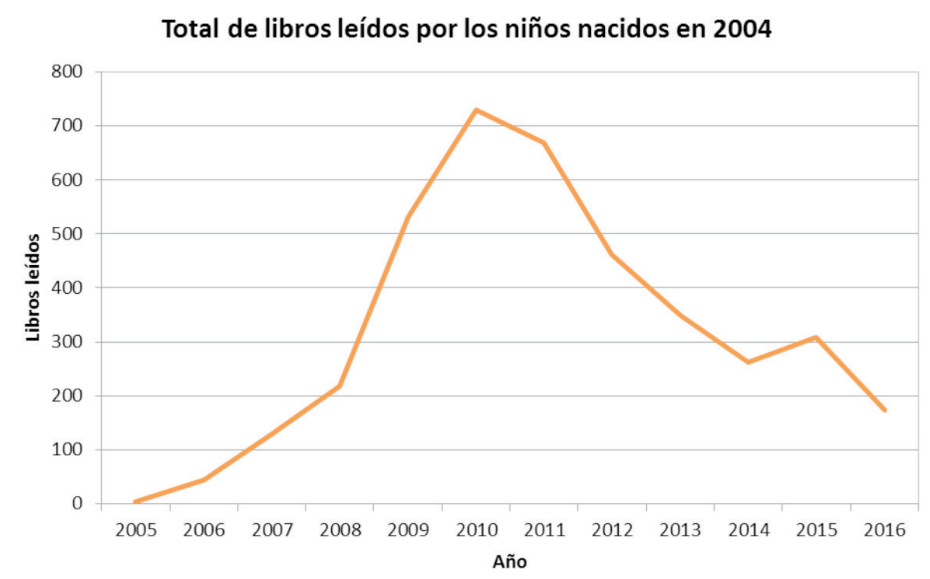

Fig 6. Percepción de una orientación a la innovación en la experiencia docente

Los resultados son también positivos, si bien encontramos una diferencia notable entre el ítem mejor valorado, puntuado con un 9,16 de promedio: "En general: Esta actividad ha sido innovadora en sus métodos y contenidos", y el peor, puntuado con un 8,43: "Sobre las charlas de expertos: han mejorado mi conocimiento últimos avances en la asignatura".

La última categoría es, como avanzábamos, algo distinta, y pretendía entender la valoración de los estudiantes ya no sobre la actividad de innovación docente, si no sobre la propia teoría del encabalgamiento y el método de análisis que se utiliza en general y en esta actividad en particular. Este grupo de ítems es especialmente relevante a la hora de redefinir tanto las actividades como el propio concepto del encabalgamiento, lo que puede ser útil para futuros trabajos filológicos. Sin embargo, no es relevante para fines pedagógicos, por lo que no lo recogemos aquí.

\subsection{Resultados obtenidos teniendo en cuenta las calificaciones finales}

En este apartado hacemos una comparativa de tres medidas estadísticas (promedio, moda y mediana) entre los estudiantes de la asignatura que se presentaron al examen sin haber realizado ni seguido la actividad de innovación docente y aquellos que sí realizaron las actividades y siguieron los vídeos de "Poesía distante". 
En la convocatoria ordinaria de junio se presentaron 137 estudiantes al examen de los 199 matriculados, es decir, casi un $70 \%$ en solo en esta primera convocatoria. Este número es muy alto en comparación con otras asignaturas de la UNED y de este grado. Las estadísticas de la UNED del 2015-2016 reflejan una tasa de evaluación para el grado en Lengua y Literatura españolas de la UNED de un 51,08\%, lo que quiere decir que solo se evalúa de media en el grado a la mitad de loes estudiantes que lo cursan, por ello, las cifras de presentación al examen en esta primera convocatoria de junio son llamativas:

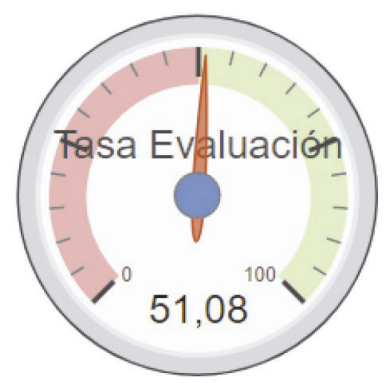

Fig 7. Tasa de evaluación del Grado en Lengua y Literatura Españolas de la UNED

La experiencia docente Distant Poetry fue seguida por 85 estudiantes, lo cual refleja un $42,71 \%$ de los estudiantes matriculados, una cifra muy significativa para los datos de una universidad a distancia. Solo 8 estudiantes que siguieron esta actividad no se presentaron al examen final, lo que es un dato de especial relevancia teniendo en cuenta las altas tasas de abandono que tiene la UNED.

Los datos permiten comparar entre los estudiantes que se presentaron al examen a) habiendo realizado la experiencia docente y b) sin haber participado en la experiencia docente:

\section{Porcentaje de libros leídos por los niños nacidos en 2004 dividido por \\ sexos}

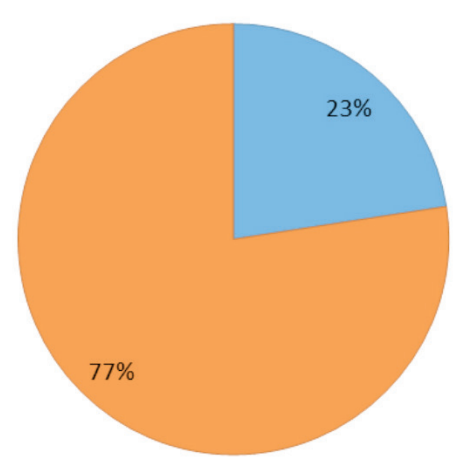

Hombres $\square$ Mujeres

Fig 8. Resultados del examen final de la asignatura desglosados en alumnos que sí siguieron la experiencia docente y los que no la siguieron 
Para medir la representatividad de los resultados hemos realizado la Prueba $U$ de Mann-Whitney (también llamada de Mann-Whitney-Wilcoxon o prueba de suma de rangos Wilcoxon), que se utiliza para comparar dos conjuntos de datos con un tipo de distribución no normalizada. Hemos comparado el conjunto de datos de las calificaciones de los estudiantes que sí realizaron la actividad (85), con el de los que no lo realizaron pero sí se presentaron al examen, es decir, se presentaron a la asignatura (52 estudiantes).

Los resultados son los siguientes:

data: notasSi and notasNo

$\mathrm{W}=3158$, p-value $=0.0002727$

alternative hypothesis: true location shift is not equal to 0

95 percent confidence interval: 0.50002171 .7999707

sample estimates:

difference in location 1.200001

El valor relevante en este tipo de prueba es el p-value, que estima las probabilidades de que ambas muestras sean iguales. En este caso es muy bajo, 0.0002727 . Hay que tener en cuenta que únicamente a partir de 0.01 se consideraría que pueden ser iguales. Estos gráficos presentan las diferencias entre los dos paquetes de datos:

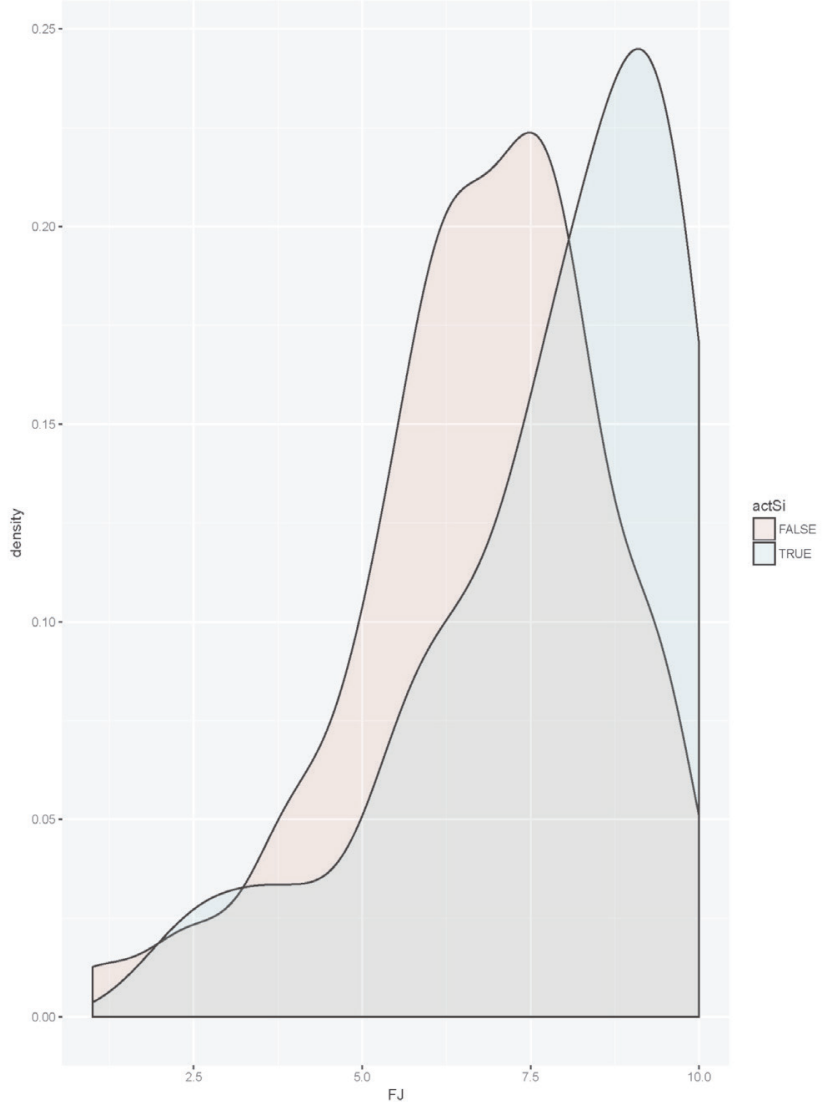

Fig 9. Resultados del examen final de la asignatura desglosados en alumnos que sí siguieron la experiencia docente y los que no la siguieron 


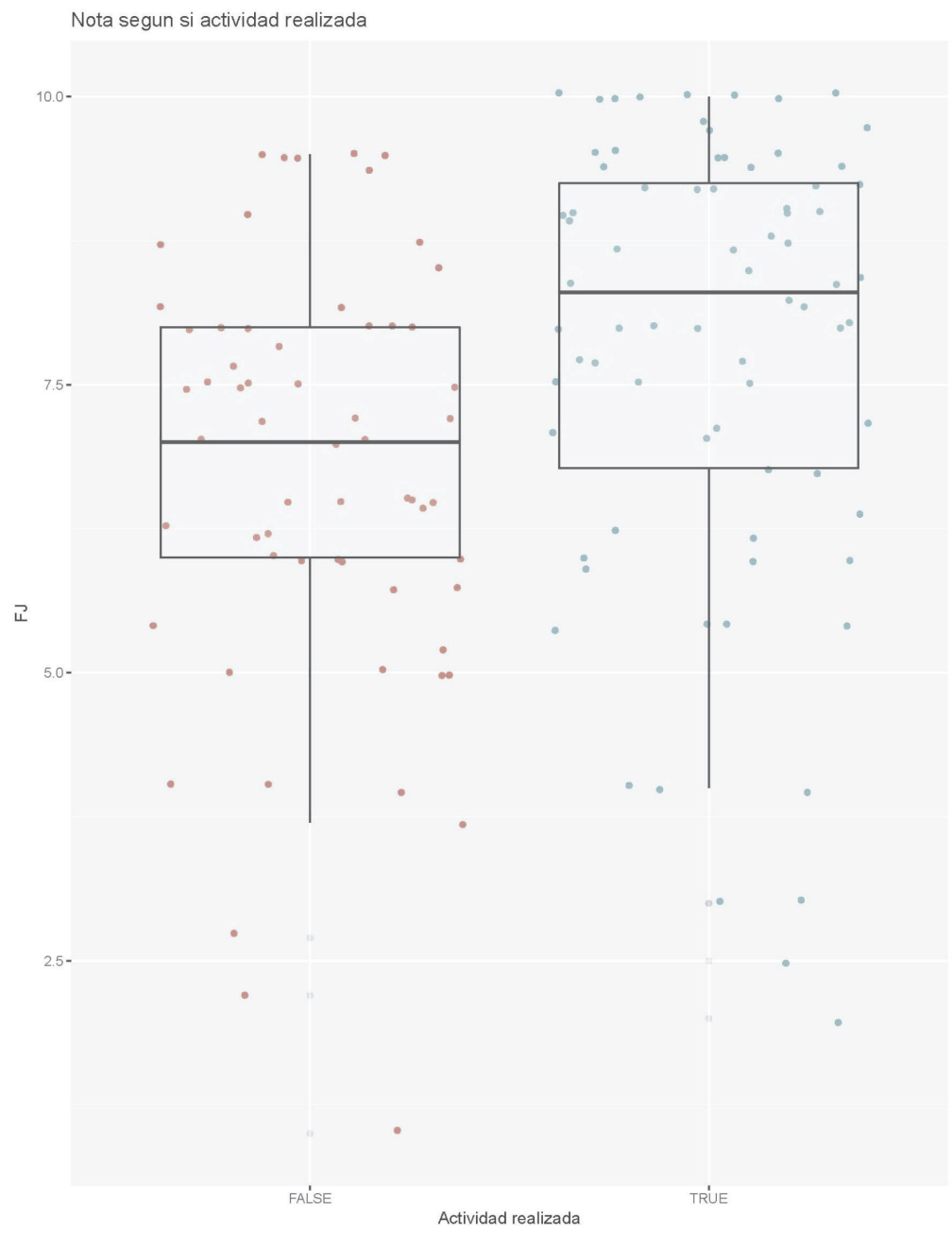

Fig 10. Gráfico de dispersión de los resultados del examen final de la asignatura desglosados en alumnos que sí siguieron la experiencia docente y los que no la siguieron

Los datos que evidencian las figuras 8, 9 y 10 muestran que los estudiantes que han seguido la actividad presentan mejores resultados significativamente. La media se incrementa en casi un punto, mientras que la moda cambia muy significativamente. La mediana también tiene más de un punto de diferencia.

\section{Discusión}

Se presentan aquí algunos de los puntos que quedan abiertos a discusión.

En primer lugar, partimos de una población de 199 estudiantes, el número de matriculados en esta asignatura. Los estudiantes que deciden seguir la actividad optativa son 85 , y de estos solo 25 han realizado el cuestionario de satisfacción. Si bien el tamaño de la muestra es pequeño queda justificado por la naturaleza altamente 
especializada y enfocada hacia la asignatura de "Estilística y métrica españolas". El curso presente está repitiéndose esta actividad, si bien con algunas modificaciones, ya que los resultados obtenidos han sido positivos.

En un título de grado en el que la el número de no presentados se cifra en torno al 50\% (ver Fig. 3 ) el hecho de que 85 estudiantes, es decir, un 42\% hayan realizado una actividad optativa con bastante carga adicional de trabajo representa ya un éxito. El número de cuestionarios respondidos es casi un $30 \%$ por lo que entendemos que podemos considerarlo significativo.

Dado que no contamos con los datos de otros años hemos comparado estos datos con los de la asignatura optativa de "Retórica" de la misma área de Teoría de la literatura y del mismo Grado. En ella encontramos que solo 14 estudiantes de 94 matriculados realizaron las actividades optativas de evaluación continua (un 14,9\%).

Se ha considerado también la posibilidad de realizar análisis estadísticos más complejos como el análisis factorial y el discriminante, pero se han descartado porque no añadían representatividad al estudio al ser la muestra pequeña y de una sola asignatura.

El resultado que correlaciona las calificaciones obtenidas en la asignatura con la realización de la experiencia docente era, de alguna manera, esperable. La realización de actividades optativas, con la carga de trabajo que implica, presupone un mayor interés y estudio, lo que mejora las calificaciones finales. En la asignatura de Retórica, también optativa, la realización de las actividades de evaluación continua optativas dejaba los siguientes datos:

- Calificación final promedio del grupo que realizó las Actividades optativas: 8,2. Mediana: 8,2.

- Calificación final promedio del grupo que no realizó las Actividades optativas: 6,94. Mediana: 7,3.

La obtención de más de un punto diferencial también en esta asignatura entre los que realizan las actividades optativas y los que no lo hacen sugiere que podría estudiarse si más que al carácter de la actividad se debe al interés de los estudiantes. Sin embargo, la experiencia realizada en "Estilística y métrica españolas" fue seguida por mucho mayor porcentaje de estudiantes, lo que ha mejorado también los resultados finales de número de presentados y sus calificaciones.

Más allá de los datos presentados cabe preguntarse en qué medida la realización de esta experiencia ha mejorado sus competencias, tanto las referidas al campo de la asignatura como las transversales tecnológicas. Si bien está claro, por los resultados obtenidos, que la actividad ha fomentado el interés de los estudiantes creemos que una interacción mayor con herramientas tecnológicas centradas en los contenidos de la asignatura podría dar mejores resultados. Con ese fin se está trabajando en una mejora del corpus de estudio, DISCO, que ofrece ya una interfaz web con diversas utilidades docentes (Ruiz Fabo, Bermúdez Sabel \& Martínez Cantón, s.f.).

Cabe dentro de esta discusión, por otra parte, una reflexión sobre la pertinencia de la enseñanza de este tipo de recursos digitales no directamente relacionados con el contenido de la asignatura. En este sentido observamos que la formación en el espacio de educación superior en competencias digitales ofrece un complicado engarce en el sistema actual de los grados. Por una parte se hace imprescindible ofrecer a los estudiantes una competencia que les permita entender y abordar los nuevos modos 
de procesamiento de la información en la sociedad de los datos, que afectan no solo a disciplinas científicas o de ciencias sociales, sino que son también omnipresentes para la cultura y las humanidades. La sociedad del siglo XXI genera y procesa cantidades ingentes de datos y es necesario formar al alumnado en el empleo de la información y la tecnología digital. Para Manuel Area Moreira (2010, p. 3) plantea cinco razones principales por las que se hace totalmente necesaria formar en competencias digitales en educación superior. Nos interesan sobre todo las dos primeras, que aquí reproducimos:

1. La producción de conocimiento en todas las áreas del saber-científico, técnico, humanístico, artístico o social- en estas últimas décadas está en permanente crecimiento exponencial y es prácticamente inabarcable. Por ello, hoy en día, un universitario debería adquirir no sólo los conceptos, teorías y conocimientos básicos de una disciplina, sino también disponer de los criterios y estrategias intelectuales para encontrar nuevas informaciones que sean valiosas para su ámbito o campo de estudio, de investigación o de actividad profesional.

2. Existen cada vez mayores y numerosas fuentes que almacenan, organizan y difunden información en formato de bibliotecas digitales, bases de datos, portales web, publicaciones electrónicas, blogs, redes sociales, etc. Por ello, es relevante que un universitario disponga de los conocimientos y habilidades de uso de estas herramientas que le permiten la búsqueda de información especializada en bases de datos bibliográficas o sitios web relevantes para su campo de conocimiento (Area Moreira, 2010, p. 3).

Estos dos puntos son básicos y deben hacer reflexionar al profesorado sobre cómo la tecnología y la sociedad de los datos han impactado en su disciplina, para reflejarlo posteriormente en las asignaturas. En universidades a distancia, como la UNED, e incluso presenciales, el manejo y el conocimiento de estas herramientas digitales se hacen además imprescindibles para el trabajo autónomo, sobre todo en entornos de e-learning.

\section{Conclusiones}

A través de esta experiencia hemos intentado introducir un giro digital en una asignatura de humanidades tradicional. La experiencia se centra en mejorar los conocimientos métricos a la vez que se incentiva la participación en las actividades de investigación universitaria, y, por lo tanto, la implicación activa del alumno, integrando elementos transversales y transdisciplinares.

Nuestra intención ha sido, además, ofrecer una visión desde las humanidades del panorama que los métodos y actividades de las HD han ido abriendo.

Se han mostrado resultados positivos en todas las categorías, con incidencia mayor en el grado de innovación en métodos y contenidos de la experiencia percibido por los estudiantes (con todos los ítems entre un 8,43 y un 9,16). Todos los ítems obtienen una valoración superior a 8 sobre 10, por lo que los estudiantes han valorado muy positivamente la experiencia. La satisfacción puede tomarse como una evidencia de la calidad en la docencia (Cantón Mayo y Téllez Martínez, 2016, p. 215). 


\section{Agradecimientos}

Se agradece a todos los profesores que han participado en la experiencia docente su colaboración, en especial a Borja Navarro y Gimena del Rio Riande.

\section{Bibliografía}

Area, Manuel (2010): “¿Por qué formar en competencias informacionales y digitales en la educación superior?", en Competencias informacionales y digitales en educación superior [monográfico en línea], en Revista de Universidad y Sociedad del Conocimiento (RUSC), 7(2). Disponible en Web: http://rusc.uoc.edu/ojs/index.php/rusc/article/view/ v7n2-area/v7n2-area [consulta: 19 de marzo 2019].

Agerri, Rodrigo, J. Bermudez y G. Rigau (2014): "IXA pipeline: Efficient and Ready to Use Multilingual NLP tools", en Proceedings of LREC 2014, the 9th International Language Resources and Evaluation Conference, Reykjavik, Iceland, 3823-3828. Disponible en Web: http:// www.lrec-conf.org/proceedings/lrec2014/pdf/775_Paper.pdf [consulta: 19 enero 2018].

Agirrezabal, Manex (2017): Automatic Scansion of Poetry, San Sebastián/Donosti, Universidad del País Vasco.

Asperti, Stefano y L. de Nigro (2012): Bibliografia Elettronica dei Trovatori - v.2.5 - 2012. Disponible en Web: http://www.bedt.it/BEdT_04_25/[consulta: 16 mayo 2019].

Birnbaum, David J. y E. Thorsen (2015): "Markup and meter: Using XML tools to teach a computer to think about versification", en Balisage: The Markup Conference. Disponible en Web: http://www.balisage.net/Proceedings/vol15/print/Birnbaum01/BalisageVol15Birnbaum01.html [consulta: 19 enero 2018].

Braun, Manuel, S. Glauch, S. y F. Kragl (s.f.): Lyrik des deutschen Mittelalters. Disponible en Web: http://www.ldm-digital.de/ [consulta: 16 mayo 2019].

Brea, Mercedes y P. Lorenzo Gradín (s.f.): MedDB. Disponible en Web: http://bernal.cirp. gal/ords/f?p=MEDDB3:2 [consulta: 16 mayo 2019].

Cantón Mayo, Isabel y S. Téllez Martínez (2016): "La satisfacción laboral y profesional de los profesores", en Revista Lasallista de Investigación, 13(1). Disponible en Web: http://repository.lasallista.edu.co:8080/ojs/index.php/rldi/article/view/996 [consulta: 19 enero 2018].

Colombi, Emanuela, L. Mondin, L. Tessarolo y A. Bacianini (s.f.): Pedecerto. Disponible en Web: http://www.pedecerto.eu/ [consulta: 16 mayo 2019].

Delente, Éliane y R. Renault (2015): “Outils et métrique: un tour d'horizon”, en Langages, $3,5-22$.

Digital Index of Middle English Verse (s.f.): Digital Index of Middle English Verse. Disponible en Web: https://www.dimev.net/ [consulta: 16 mayo 2019].

Forcada, M Mikel L., M. Ginestí-Rosell, J. Nordfalk, J. O'Regan, S. Ortiz-Rojas, J. A. PérezOrtiz, ... F. M. Tyers (2011): "Apertium: a free/open-source platform for rule-based machine translation", en Machine translation, 25(2), 127-144.

García González, Ramón (Ed.) (2006a): Sonetos del siglo XIX, Alicante, Biblioteca Virtual Miguel de Cervantes. Disponible en Web: http://www.cervantesvirtual.com/obra/sonetos-del-siglo-xix--0/http://www.cervantesvirtual.com/obra/sonetos-del-siglo-xv-alxvii--0/ [consulta: 19 enero 2018].

García González, Ramón (Ed.) (2006b): Sonetos del siglo XV al XVII, Alicante, Biblioteca Virtual Miguel de Cervantes. Disponible en Web: http://www.cervantesvirtual.com/obra/ sonetos-del-siglo-xv-al-xvii--0/ [consulta: 19 enero 2018]. 
Gervás, Pablo (2000a): A Logic Programming Application for the Analysis of Spanish Verse, en Computational Logic-CL 2000, Berlin, Springer Berlin Heidelberg, 13301344.

Gervás, Pablo (2000b): "Wasp: Evaluation of different strategies for the automatic generation of Spanish verse", en Proceedings of the AISB-00 symposium on creative \& cultural aspects of AI, 93-100. Disponible en Web: https://www.researchgate.net/profile/Pablo_Gervas/publication/228609235_Wasp_Evaluation_of_different_strategies_for the automatic_generation_of_spanish_verse/links/00b4952aada6407047000000.pdf [consulta: 19 enero 2018].

Gervás, Pablo (2015): “Tightening the Constraints on Form and Content for an Existing Computer Poet", en AISB Convention 2015. Disponible en Web: http://eprints.sim.ucm. es/37000/ [consulta: 19 enero 2018].

Gold, Matthew K. (Ed.) (2012): Debates in the Digital Humanities, Univerity of Minnesota Press.

Gonzalez-Blanco, Elena, C. I. Martínez Cantón y G. Río Riande (2015): “El Laboratorio de Innovación en Humanidades Digitales y la redefinición del perfil del humanista y la academia en el siglo XXI", en Las humanidades digitales desde Argentina. Tecnologías, culturas, saberes, L. Cantamutto, G. Río Riande y G. Striker (Eds.), 59-68. Buenos Aires, Editorial de la Facultad de Filosofía y Letras Universidad de Buenos Aires. Disponible en Web: http://e-spacio.uned.es/fez/view/bibliuned:363-Egonzalez2 [consulta: 19 enero 2018].

González-Blanco García, Elena (s.f.): Remetca. Dispoible en Web: http://www.remetca.uned. es [consulta: 19 enero 2018].

González-Blanco García, Elena (2013): “Actualidad de las Humanidades Digitales y un ejemplo de ensamblaje poético en la red", en Cuadernos hispanoamericanos, 761, 53-68.

Horváth, Iván (2001): RPHA. Disponible en Web: http://rpha.elte.hu/ [consulta: 16 mayo 2019].

Huber, Alexander (s.f.): Eighteenth-Century Poetry Archive. Disponible en Web: https:// www.eighteenthcenturypoetry.org/ [consulta: 16 mayo 2019].

Le Nouveau Naetebus (s.f.). Disponible en Web: http://nouveaunaetebus.elte.hu/ [consulta: 16 mayo 2019].

Lloberes, Marina, I. Castellón y L. Padró (2010): "Spanish FreeLing Dependency Grammar", en LREC, 10, 693-699. Disponible en Web: http://www.lrec-conf.org/proceedings/ 1rec2010/pdf/562_Paper.pdf [consulta: 19 enero 2018].

Maroto Sánchez, Antonio (2007): "El uso de las nuevas tecnologías en el profesorado universitario", en Pixel-Bit: Revista de medios y educación, 30, 61-72.

Mir Acebrón, Anna (2008): "Las competencias transversales en la Universidad Pompeu Fabra: la visión de los docentes y estudiantes de segundo ciclo", en REDU: Revista de Docencia Universitaria, 1, 2.

Moretti, Franco (2013): Distant reading, London \& New York, Verso Books.

Navarro-Colorado, Borja (2017): “A metrical scansion system for fixed-metre Spanish poetry", en Digital Scholarship in the Humanities, 33(1), 112-127. Disponible en Web: https://doi.org/10.1093/llc/fqx009 [consulta: 19 enero 2018].

Navarro-Colorado, Borja, M. R. Lafoz y N. Sánchez (2016): “Metrical Annotation of a Large Corpus of Spanish Sonnets: Representation, Scansion and Evaluation”, en Proceedings of the Tenth International Conference on Language Resources and Evaluation, Portoroz, Slovenia. Portorož, Slovenia, 4630-4634. Disponible en Web: http:/www.lrec-conf.org/ proceedings/lrec2016/pdf/453_Paper.pdf [consulta: 19 enero 2018]. 
Nederlandse Liederenbank (s.f.). Nederlandse Liederenbank Project. Disponible en web: http://www.liederenbank.nl/ [consulta: 16 mayo 2019].

Padró, Lluís y E. Stanilovsky (2012): "Freeling 3.0: Towards wider multilinguality", en LREC2012. Disponible en Web: https://upcommons.upc.edu/handle/2117/15986 [consulta: 19 enero 2018].

Parkinson, Stephen (s.f.): The Oxford Cantigas de Santa Maria database. Disponible en Web: http://csm.mml.ox.ac.uk/ [consulta: 16 mayo 2016].

Rauner, Erwin (Ed. online) (s.f.): Analecta Hymnica Digitalia. Disponible en Web: http:// webserver.erwin-rauner.de/crophius/Analecta_conspectus.htm [consulta: 16 mayo 2019].

Río Riande, Gimena, E. González-Blanco y C. I. Martínez Cantón, C. I. (2015): “Making visible the invisible: metrical patterns, contrafacture and compilation in a Medieval Castilian Songbook", en DH 2015: Global Digital Humanities, Sydney, Australia. Disponible en Web: http://dh2015.org/abstracts/ [consulta: 19 enero 2018].

Rojas Castro, Antonio (2013): "El mapa y el territorio. Una aproximación histórico-bibliográfica a la emergencia de las Humanidades Digitales en España”, en Caracteres. Estudios culturales y críticos de la esfera digital. Disponible en Web: http://revistacaracteres. net/revista/vol2n2noviembre2013/el-mapa-y-el-territorio/ [consulta: 19 enero 2018].

Ruiz, Pablo, H. Bermúdez Sabel y C. I. Martínez Cantón (s.f.): DISCO. Disponible en Web: http://prf1.org/DISCO/ [consulta: 16 mayo 2019].

Ruiz, Pablo, C. I. Martínez Cantón, T. Poibeau y E. González-Blanco (2017): “Enjambment Detection in a Large Diachronic Corpus of Spanish Sonnets", en Proceedings of the Joint SIGHUM Workshop on Computational Linguistics for Cultural Heritage, Social Sciences, Humanities and Literature, Association for Computational Linguistics, 27-32.

Ruiz Fabo, Pablo, H. Bermúdez Sabel, C. I. Martínez Cantón, E. González-Blanco y B. Navarro Colorado (2018). "The Diachronic Spanish Sonnet Corpus (DISCO): TEI and Linked Open Data Encoding, Data Distribution and Metrical Findings", en DH 2018. Book of abstracts, México DF, 486-489. Disponible en Web: https://dh2018.adho.org/ the-diachronic-spanish-sonnet-corpus-disco-tei-and-linked-open-data-encoding-datadistribution-and-metrical-findings/ [consulta: 19 febrero 2019].

Schreibman, Susan, R. Siemens y J. Unsworth (Eds.) (2004): A Companion to Digital Humanities, Malden, MA: Wiley-Blackwell.

Schreibman, Susan, R. Siemens y J. Unsworth, J. (2016): A New Companion to Digital Humanities (Edición: 2 Rev ed.), Chichester, West Sussex, Blackwell Companions to Literature and Culture.

Stella, Francesco y S. Barrett (Coords.) (s.f.): Corpus Rhythmorum Musicum (saec. IV-IX) - I. Disponible en Web: http://www.corimu.unisi.it/ [consulta: 16 mayo 2019].

The Last Song of the Troubadours (s.f.): The Last Song of the Troubadours. Disponible en Web: http://www.lastsongtroubadours.eu/ [consulta: 16 mayo 2019].

UNED. (2014): Memoria de verificación del gítulo de Grado en Lengua y Literatura Españolas por la Universidad Nacional de Educación a Distancia. Disponible en Web: https:// descargas.uned.es/publico/pdf/guias/6401/MEMORIA_VERIFICACION_6401.pdf [consulta: 19 enero 2019].

UNED, CONICET y UNAM. (s.f.): Revista de Humanidades Digitales. Disponible en Web: http://revistas.uned.es/index.php/RHD/index [consulta: 16 mayo 2019].

University of Southampton (s.f.): CPI Conductus: Thirteenth-Century Music and Poetry. Disponible en Web: http://catalogue.conductus.ac.uk/ [consulta: 16 mayo 2019].

Wills, Tarris (s.f.): Skaldic Project. Disponible en Web: https://skaldic.abdn.ac.uk/db.php [consulta: 16 mayo 2019]. 